\title{
Improved stability analysis of networked control systems under asynchronous sampling and input delay
}

\author{
Wenjuan Jiang * Alexandre Seuret* \\ * NeCS Team, Department of Automatic Control, \\ GIPSA-Lab-CNRS UMR 5216, Grenoble, France \\ (e-mail: wenjuan.jiang,alexandre.seuret@gipsa-lab.inpg.fr).
}

\begin{abstract}
This article presents a novel approach to assess the stability of linear systems with delayed and sampled-data inputs. It proposes an extension of existing results on the stability of sampled-data systems to the case where a delay is introduced in the control loop. The method is based on a continuoustime modelling of the systems together with the discrete-time Lyapunov theorem, which provides easy tractable sufficient conditions for asymptotic stability. Those conditions cope the problem of stability under asynchronous samplings and time-varying delays. The period and delay-dependent conditions are expressed using computable linear matrix inequalities. Several examples show the efficiency of the stability criteria.
\end{abstract}

Keywords: Sampled-Data systems, Lyapunov-Krasovskii functionals, Linear systems, Delay systems.

\section{INTRODUCTION}

In the last decades, a large attention has been taken to Networked Control Systems (NCS) (see [Hespanha et al., 2007], or [Zampieri, 2008]). Such systems are controlled systems containing several distributed plants which are connected through a communication network. In such applications, one has to check the robustness of a control law with respect to the additional dynamics introduced by the communication networks. Among these dynamics, this article focuses on the influence of transmission delay and asynchronous samplings. The transmission of a data packet through a network can not be achieved instantaneously. Transmission delays are unavoidably introduced. Those delays may lead to instability [Richard, 2003]. On the other hand, a heavy temporary load of computation in a control processor can corrupt the sampling period of a certain controller. We can also imagine a situation where the sampling period is scheduled in the control design in order to avoid this load. In both cases, the variations of the sampling period will also affect the stability properties. It is thus an important issue to develop robust stability criteria with respect to transmission delays and asynchronous samplings.

Sampled-data systems have extensively been studied in the literature [Chen and Francis, 1995, Fridman et al., 2004, Fujioka, 2009, Zhang and Branicky, 2001, Zhang et al., 2001] and the references therein. It is now reasonable to design controllers which guarantee the robustness of the solutions of the closed-loop system under periodic samplings. However the case of asynchronous samplings still leads to several open problems such that the guarantee of stability whatever the sampling period lying in an interval. Recently, several articles drive the problem of time-varying periods based on a discretetime approach, [Yue et al., 2008, Oishi and Fujioka, 2009, Hetel et al., 2006]. Note that the discrete-time approaches do

\footnotetext{
* This research is supported by FeedNetBack project, FP7- ICT-2007-2: http://www.feednetback.eu/.
}

not fit with the case of uncertain systems or systems with time-varying parameters. Recent papers considered the modelling of continuous-time systems with sampled-data control in the form of continuous-time systems with delayed control input. In [Fridman et al., 2004], a Lyapunov-Krasovskii approach is introduced. Improvements are provided in [Fujioka, 2009, Mirkin, 2007], using the small gain theorem and in [Naghshtabrizi et al., 2008] based on the analysis of impulsive systems. These approaches are very relevant because they deal with time-varying sampling periods and with uncertain systems (see [Fridman et al., 2004] and [Naghshtabrizi et al., 2008]). Nevertheless, these sufficient conditions are still conservative. This means that the conditions obtained by continuous-time approaches are not able to guarantee asymptotic stability whereas the system is stable. Recently several authors [Fridman, 2010, Liu and Fridman, 2009a, Seuret, 2009, 2010] refine those approaches and obtain tighter conditions.

When transmission delays are introduced in the control loop, the problem becomes more complex. It is indeed well known that delays require a more accurate analysis since the timedelay systems are of infinite dimension [Gu et al., 2003, Richard, 2003]. Several articles have been provided to cope with the stability of NCS under sampling and transmission delays. In [Fridman et al., 2004, Liu and Fridman, 2009b, Naghshtabrizi et al., 2010], stability conditions of systems under asynchronous sampling and transmission delays are presented. However those conditions are still conservative and require improvements. In the present article, we provide a novel method to assess asymptotic stability of such systems. The conditions are presented as an extension of [Seuret, 2010] to the case of time-varying transmission delays. This problem of NCS proposed here is hybrid since we consider a continuous time model of the plant and a discrete-time communication. Thus another important improvement presented here consists in employing the discrete-time Lyapunov theorem to continuoustime modelling of sampled-data systems with delays. The main 


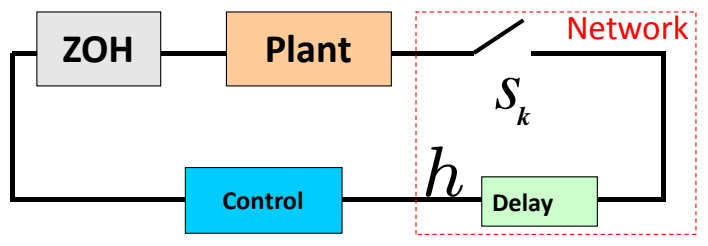

Fig. 1. Control loop of Networked control systems under transmission and sampling delays

idea in this paper is to consider separately the two types of delays. This method provides a larger upper-bound of the allowable sampling period than the existing ones (based on the continuous-time approach).

This article is organized as follows. The next section formulates the problem and presents a lemma that will be used in the sequel. Section 3 exposes the novel stability criteria based on the discrete-time Lyapunov theorem. Then, in Section 4, asymptotic stability criteria for sampled-data systems are exposed to cope with time-varying input delays. Some examples are provided in Section 5 which shows the efficiency of the method.

Notations. Throughout the article, for a n-dimensional state vector $x$ and a non-negative delay $h, x_{t}$ denotes a function such that $x_{t}(\theta)=x(t-\theta)$ for all $\theta \in[-h, 0]$. The notation $\mathbb{K}_{h}$ stands for the set of the functions from $[-h, 0]$ to $\mathbb{R}^{n}$. The sets $\mathbb{R}^{+}, \mathbb{R}^{n \times n}$ and $\mathbb{S}^{n}$ denote respectively the set of positive scalar, the set of $n \times n$ matrices and the set of symmetric matrices of $\mathbb{R}^{n \times n}$. The superscript ' $T$ ' stands for the matrix transposition. The notation $P>0$ for $P \in \mathbb{S}^{n}$ means that $P$ is positive definite, then the set of symmetric definite positive matrices is denoted as $\mathbb{S}_{+}^{n}$. The symbols $I$ and 0 represent the identity and the zero matrices of appropriate dimension. Recall that a function $\gamma: \mathbb{R}^{+} \rightarrow \mathbb{R}^{+}$is a $\mathcal{K}$-function if it is continuous, strictly increasing and $\gamma(0)=0$. A function $\gamma^{\prime}$ belongs to the set $\mathcal{K}_{\infty}$ if $\gamma^{\prime} \in \mathcal{K}$ and $\gamma(s) \rightarrow \infty$ as $t \rightarrow \infty$. A function $\beta: \mathbb{R}^{+} \times \mathbb{R}^{+} \rightarrow \mathbb{R}^{+}$is a $\mathcal{K} \mathcal{L}$-function if, for each fixed $t \geq 0$, the function $\beta(\cdot, t)$ is a $\mathcal{K}$-function, and for each fixed $s \geq 0$, the function $\beta(s, \cdot)$ is decreasing and $\beta(s, t) \rightarrow 0$ as $t \rightarrow \infty$. For any matrices $A_{i}, A_{j}, A_{i j}=A_{i}-A_{j}$.

\section{PROBLEM FORMULATION}

\subsection{System definition}

Consider the linear system with a sampled and delayed input as shown in Figure 1:

$$
\dot{x}(t)=A x(t)+B u(t),
$$

where $x \in \mathbb{R}^{n}$ and $u \in \mathbb{R}^{m}$ represent the state variable and the input vector. The matrices $A$ and $B$ are constant and of appropriate dimension. As in the situation of networked control systems, the control input $u$ is affected by the networked communication. In this paper it is only assumed that the network induces a time-varying transmission delay $h$ and a sampling of the transmitted signal. We are looking for a piecewise-constant static state-feedback control law:

$$
u(t)=K x\left(s_{k}\right), s_{k}+h\left(s_{k}\right) \leq t<s_{k+1}+h\left(s_{k+1}\right)
$$

where $0=s_{0}<s_{1}<\ldots<s_{k}<\ldots$ represent the sampling instants. The sequence of $\left\{s_{k}\right\}_{k \geq 0}$ is strictly increasing and goes to infinity as $k$ increases. The transmission delay $h(t)$ is assumed to be constant or time-varying and such that

$$
\forall t, \quad h(t) \in\left[h_{1} h_{2}\right], \quad \epsilon_{1} \leq \dot{h}(t) \leq \epsilon_{2}<1
$$

where $0 \leq h_{1}<h_{2}$ and $\epsilon_{1}<\epsilon_{2}$. In order to simplify the notation, $h_{k}=h\left(s_{k}\right)$ is introduced. Denote $t_{k}=s_{k}+h_{k}$. These instants $t_{k}$ represent the instants where the control input is updated, which refers to the actuation instants. Our objective is to ensure the stability of the system together with a statefeedback controller of the form

$$
u(t)=K x\left(t_{k}-h_{k}\right), t_{k} \leq t<t_{k+1} .
$$

where the gain $K$ in $\mathbb{R}^{n \times m}$ is given. Assume that there exists a positive scalar $T$ such that the difference between two successive sampling instants $T_{k}=s_{k+1}-s_{k}$ satisfies

$$
\forall k \geq 0, \quad 0 \leq T_{1} \leq T_{k} \leq T_{2} .
$$

Then the actuation instants $t_{k}$ of the control input satisfies

$$
\bar{T}_{k}=t_{k+1}-t_{k}=s_{k+1}-s_{k}+h_{k+1}-h_{k} .
$$

In order to keep a chronological order of the control values, the value $\bar{T}_{k}$ is necessarily positive, which leads to the condition

$$
\forall k \geq 0, \quad T_{k}>h_{k}-h_{k+1} .
$$

Several authors investigated in guaranteeing the stability of such systems. In Fridman et al. [2004], a continuous-time approach to model sampled-data systems was developed. It allows assimilating sampling effects as the ones of a particular delay. Substituting (3) into (1), we obtain the following closedloop system:

$$
\begin{aligned}
& \dot{x}(t)=A x(t)+A_{d} x\left(t_{k}-h_{k}\right), \\
& \tau(t)=t-t_{k}, t_{k} \leq t<t_{k+1} .
\end{aligned}
$$

where $A_{d}=B K$ and where the continuous delay function corresponds to the continuous extension of the $h_{k}$ within a sampling period. From (4), it follows that $\tau(t) \leq T$ since $\tau(t) \leq t_{k+1}-t_{k}$. We will further consider (5) as a linear system with uncertain and bounded delay $\delta(t)=t-t_{k}+h_{k}$. An example of such delays is presented in Figure 2.

In Fridman et al. [2004] or Millán et al. [2009], the authors propose an aggregated delay formulation. They develop stability criteria which take into account the delay $\delta$. However they did not consider the different natures of the transmission and the sampling delay. More especially the additional characteristic of sampled delay which is $\dot{\tau}=1$ has not been included and thus leads to conservative conditions.

In this paper, the aggregated delay $\delta$ representing the effect of the transmission and the sampling delays is split into two parts. The main idea is to consider separately the two types delays. This allows having $\delta$ greater than the upper bound $h_{2}$. In this paper, a novel method to assess stability of systems subject to this type of delay is proposed. The present article establishes a novel approach to cope with the stability analysis of continuous-time systems under delayed and sampled inputs. This method is based on the discrete-time Lyapunov Theorem and leads to less conservative necessary conditions. Those conditions concern a class of functionals which are not required to be of the Lyapunov-Krasovskii type. For the sake of simplicity, the notation $\boldsymbol{\tau}, \boldsymbol{h}$ and $\dot{h}$ stand for the time-varying sampling delay $\tau(t)$, the time-varying transmission delay $h(t)$ and its time-varying derivative $\dot{h}(t)$.

Integrating the differential equation (5) with the control law (3) over the interval $\left[t_{k}, s\right]$, for any instant $s$ in $\left[t_{k}, t_{k+1}\right]$, the dynamics of the system satisfies 


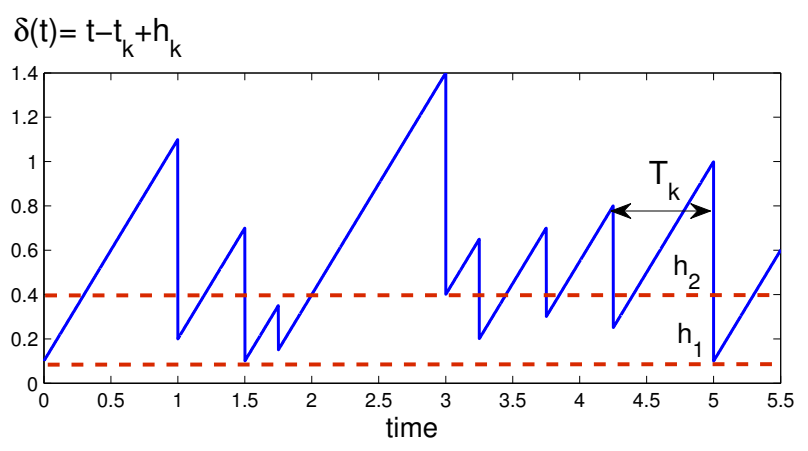

Fig. 2. Examples of a delay generated by a transmission delay $h_{k}$ bounded by $h_{1}$ and $h_{2}$ and an asynchronous sampling of periods $T_{k}$

$\forall s \in\left[t_{k}, t_{k+1}\right], x(s)=\tilde{A}(\tau(s)) x\left(t_{k}\right)+\tilde{A}_{d}(\tau(s)) x\left(t_{k}-h_{k}\right)$, $\forall \tau \in[0, \bar{T}], \tilde{A}(\tau)=e^{A \tau}, \quad \tilde{A}_{d}(\tau)=\int_{0}^{\tau} e^{A(\tau-\theta)} d \theta B K$.

This equality leads naturally to the introduction of a novel notation. Define the function for all integer $k, \chi_{k}^{h_{2}}:\left[0, T_{k}\right] \times$ $\left[-h_{2}, 0\right] \rightarrow \mathbb{R}^{n}$ such that for all $\tau$ in $\left[0, T_{k}\right]$ and all $\theta$ in $\left[-h_{2}, 0\right], \chi_{k}(\tau, \theta)=x\left(t_{k}+\tau+\theta\right)$. The set $\mathbb{K}_{\bar{T}}^{h_{2}}$ represents the set of functions defined by $\chi_{k}^{h_{2}}$ as the set of continuous functions from $[0, \bar{T}] \times\left[-h_{2}, 0\right]$ to $\mathbb{R}^{n}$ where $\bar{T}$ is the upperbound of the $T_{k}$ in (4).

\subsection{Preliminary lemma}

In order to clarify the presentation, the following lemma on a property of convex linear matrix inequalities is stated.

Lemma 1. [Naghshtabrizi et al., 2008] Consider three matrices $X_{1}, X_{2}$ and $X_{3} \in \mathbb{S}^{n}$ and a time-varying parameter $\lambda$ : $\mathbb{R}^{+} \rightarrow\left[\lambda_{m} \lambda_{M}\right]$, for some given $\lambda_{m}$ and $\lambda_{M}$. If the following inequality is guaranteed

$$
\forall t \geq 0, \quad X_{1}+\left(\lambda_{M}-\lambda(t)\right) X_{2}+\left(\lambda(t)-\lambda_{m}\right) X_{3}<0,
$$

then, it is equivalent to

$$
X_{1}+\left(\lambda_{M}-\lambda_{m}\right) X_{2}<0, X_{1}+\left(\lambda_{M}-\lambda_{m}\right) X_{3}<0 .
$$

\section{MAIN RESULT}

This section is motivated by the difference between the discrete and continuous-time Lyapunov Theorems. As the problem of sampled-data systems is at the boundary of the discrete and the continuous-time theories, it is important to put in clear the difference between them. More especially, the main idea of this section consists in developing a novel stability criterion for systems, taken in a continuous-time model, using the discretetime Lyapunov Theorem.

Theorem 1. Let $V: \mathbb{K}^{h_{2}} \rightarrow \mathbb{R}^{+}$be a functional for which there exist real numbers $0<\mu_{1}<\mu_{2}$ and $p>0$ such that

$$
\forall\left(x_{t}\right) \in \mathbb{K}, \quad \mu_{1}\left|x_{t}(0)\right|^{p} \leq V\left(x_{t}\right) \leq \mu_{2}\left|x_{t}\right|^{p} .
$$

The two following statements are equivalent.

(i) $\forall k \geq 0, \quad \Delta V(k)=V\left(x_{t_{k+1}}\right)-V\left(x_{t_{k}}\right)<0$;

(ii) There exists a continuous functional $\mathcal{V}: \mathbb{R} \times \mathbb{K}_{\bar{T}}^{h_{2}} \rightarrow \mathbb{R}$, differentiable over all intervals of the form $\left[t_{k} t_{k+1}\right.$ [ which satisfies

$$
\forall k \geq 0, \quad \mathcal{V}\left(T_{k}, \chi_{k}^{h_{2}}\right)=\mathcal{V}\left(0, \chi_{k}^{h_{2}}\right)
$$

and such that, for all $k>0$ and for all $t$ in $\left[t_{k} t_{k+1}\right]$, the following inequality holds

$$
\begin{gathered}
\mathcal{W}\left(\tau(t), \chi_{k}^{h_{2}}\right)<0 \\
\text { where } \mathcal{W}\left(\tau(t), \chi_{k}^{h_{2}}\right)=\frac{d}{d t}\left\{\left[V\left(x_{t}\right)+\mathcal{V}\left(\tau(t), \chi_{k} \chi_{k}^{h_{2}}\right)\right]\right\} .
\end{gathered}
$$

Moreover, if one of these two statements is satisfied, the solutions of system (1) with the control law (3) are asymptotically stable.

Proof. Consider a positive integer $k$ and $t \in\left[t_{k}, t_{k+1}\right]$. Assume (ii) is satisfied. Integrating $\mathcal{W}_{\alpha}$ over the interval $\left[t_{k} t_{k+1}[\right.$ and assuming that (10) holds, this directly implies $\Delta_{\alpha} V(k)<0$ and that $(i)$ holds.

Assume now that $(i)$ is satisfied. Inspired by Lemma 2 in Peet et al. [2009], consider the functional $\mathcal{V}\left(\tau, \chi_{k}^{h_{2}}\right)=-V\left(x_{t}\right)+$ $\tau / T_{k} \Delta V(k)$. Indeed, $\mathcal{V}$ is a functional since it is expressed with respect to $\Delta V(k)$ which depends on the function $\chi_{k}^{h_{2}}(0, \theta)=$ $x_{t_{k}}(\theta), \chi_{k}^{h_{2}}\left(T_{k}, \theta\right)=x_{t_{k+1}}(\theta)$ and $\chi_{k}(\tau, \theta)=x_{t}(\theta)$ for all $t \in$ $\left[t_{k}, t_{k+1}\right]$. By simple computations, it is easy to obtain that this functional satisfies (10) and that $\mathcal{W}\left(\tau, \chi_{k}\right)=\Delta V(k)$. Thus, $\mathcal{W}$ has the same sign as $\Delta V(k)$. This proves the equivalence between $(i)$ and $(i i)$.

From the discrete-time Lyapunov theorem, the equilibrium of the discrete-time system is asymptotically stable.

The end of the proof consists in ensuring that the solutions of the continuous-time system are not diverging within a sampling period. Consider any $s \in\left[t_{k}, t_{k+1}\right]$. From (9), it follows that

$$
V\left(x_{s}\right)<\lambda_{2}\left|x_{s}\right|
$$

From (6), the following equality holds

$$
x(s)=\tilde{A}(\tau(s)) x\left(t_{k}\right)+\tilde{A}_{d}(\tau(s)) x\left(t_{k}-h_{k}\right),
$$

where $\tilde{A}$ and $\tilde{A}_{d}$ are given in (6). Since those matrix functions are considered only on $\tau(s) \in\left[0, T_{\max }\right]$ and continuous over this interval, it is clear that there exist a constant parameter $\lambda^{*}$ such that $|x(s)| \leq \lambda^{*}\left|x_{t_{k}}\right|$. Since $\left|x_{t_{k}}\right|$ is converging to zero as $t_{k}$ tends to infinity, it is clear that $x(s)$ also converges to zero as $t_{k}$ tends to infinity.

The statement of Theorem 1 is finally simple. However the consequences on the functional $\mathcal{V}$ are relevant. No conditions on the positive definiteness of $\mathcal{V}$ are introduced. The positivity requirements to ensure stability is on the functional $V$ and (10).

Knowing that $V\left(x_{t_{k+1}}\right)-V\left(x_{t_{k}}\right)=\int_{t_{k}}^{t_{k+1}} \dot{V}\left(x_{s}\right) d s$, the objective consists in ensuring that this integral is negative definite. In [Peet et al., 2009], it was shown that there is an equivalence between this condition and the existence of a function $\dot{\mathcal{V}}$, such that $\int_{t_{k}}^{t_{k+1}} \dot{\mathcal{V}}=0$ and $\dot{V}+\dot{\mathcal{V}}<0$. Thus the objective is to find appropriate functionals $\mathcal{V}$ such that $\mathcal{W}<0$.

In the following, a theorem provides sufficient conditions to ensure (11) in the case of time-varying delay $h$.

\section{STABILITY CRITERIA}

Consider system (5) under an asynchronous sampling (i.e. $T_{k}$ is time-varying) and a time-varying delay $\boldsymbol{h}$ satisfying (2). The following theorem is proposed:

Theorem 2. For given $0 \leq T_{1}<T_{2}$ and $0 \leq h_{1}<h_{2}$, consider an asynchronous sampling and a time-varying delay $\boldsymbol{h}$ which satisfy (2) and (4). Assume that there exist, for $p=1,2,3$ 
and for $l=1,2,3,4$, the matrices $Q_{p}$ and $R_{l} \in \mathbb{S}_{+}^{n}, P$ and $U \in \mathbb{S}_{+}^{2 n}, S_{1}$ and $X \in \mathbb{S}^{2 n}$ and three matrices $S_{2} \in \mathbb{R}^{2 n \times 2 n}$, $Y_{1}, Y_{2} \in \mathbb{R}^{2 n \times n}, Y_{3} \in \mathbb{R}^{5 n \times 2 n}$, that satisfy for $i=1,2$ and $j=1,2$

$$
\begin{gathered}
{\left[\begin{array}{cc}
\Pi_{1}\left(h_{j}\right)+\bar{T}_{i}\left(N_{2}^{T} X N_{2}+\Pi_{2}\right) & h_{21} N_{1} Y_{j} \\
* & -h_{21} \Pi_{3 j}
\end{array}\right]<0,} \\
{\left[\begin{array}{ccc}
\Pi_{1}\left(h_{j}\right)-\bar{T}_{i} N_{2}^{T} X N_{2} & \bar{T}_{i} N_{3} Y_{3} & h_{21} N_{1} Y_{j} \\
* & -\bar{T}_{i} U & 0 \\
* & * & -h_{21} \Pi_{3 j}
\end{array}\right]<0,} \\
Q_{23}=Q_{2}-Q_{3}>0
\end{gathered}
$$

where $\bar{T}_{1}=\max \left(0, T_{1}-h_{21}\right), \bar{T}_{2}=T_{2}+h_{21}$ and

$$
\begin{aligned}
\Pi_{1}\left(h_{i}\right) & =2 N_{1}^{T} P N_{0}-N_{12}^{T} S_{1} N_{12}-2 N_{2}^{T} S_{2} N_{12} \\
& +M_{1}^{T} Q_{1} M_{1}-\left(1-\epsilon_{2}\right) M_{2}^{T} Q_{23} M_{2} \\
& -M_{3}^{T} Q_{12} M_{3}-M_{4}^{T} Q_{3} M_{4}+M_{0}^{T} \tilde{R}\left(h_{i}\right) M_{0} \\
& -1 / h_{1} M_{13}^{T}\left(\left(1-\epsilon_{2}\right) R_{2}+R_{3}\right) M_{13} \\
& -1 /\left(1-\epsilon_{1}\right) M_{5}^{T} R_{1} M_{5}-2 N_{1} Y_{1} M_{24} \\
& +2 N_{1} Y_{2} M_{23}-2 N_{3} Y_{3} N_{12}, \\
\Pi_{2}= & N_{0}^{T}\left(U N_{0}+2 S_{1} N_{12}+2 S_{2}^{T} N_{2}\right), \\
\Pi_{31}= & R_{3}+R_{4}, \\
\Pi_{32}= & \left(1-\epsilon_{2}\right) R_{2}+R_{3}+R_{4}, \\
\tilde{R}\left(h_{i}\right)= & R_{1}+h_{i} R_{2}+h_{2} R_{3}+h_{21} R_{4} .
\end{aligned}
$$

The constant matrices $M_{i} \in \mathbb{R}^{n \times 7 n}$, for $l=0, \ldots, 7$ are such that

$$
\begin{aligned}
& {\left[M_{1}^{T} M_{2}^{T} \ldots M_{7}^{T}\right]=I} \\
& M_{0}=A_{0} M_{1}+A_{d} M_{7} \\
& N_{0}=\left[M_{0}^{T} M_{5}^{T}\right]^{T}, N_{1}=\left[M_{1}^{T} M_{2}^{T}\right]^{T}, N_{2}=\left[M_{6}^{T} M_{7}^{T}\right]^{T}, \\
& N_{3}=\left[M_{1}^{T} M_{2}^{T} M_{5}^{T} M_{6}^{T} M_{7}^{T}\right]^{T}
\end{aligned}
$$

The matrices $M_{i j}=M_{i}-M_{j}, N_{i j}=N_{i}-N_{j}, i, j \in 1 \ldots 7$ refer to the notation at the end of the section introduction. System (5) is thus asymptotically stable for the sampling period $T$ and the time-varying input delay $h$.

Proof. Consider the functional:

$$
\begin{aligned}
& V\left(t, x_{t}, \dot{x}_{t}\right)=z^{T}(t) P z(t)+\int_{t-h_{1}}^{t} x^{T}(s) Q_{1} x(s) d s \\
& +\int_{t-\boldsymbol{h}}^{t-h_{1}} x^{T}(s) Q_{2} x(s) d s+\int_{t-h_{2}}^{t-\boldsymbol{h}} x^{T}(s) Q_{3} x(s) d s \\
& +\int_{t-\boldsymbol{h}}^{t} \dot{x}^{T}(s)\left(R_{1}+(\boldsymbol{h}-t+s) R_{2}\right) \dot{x}(s) d s \\
& +\int_{-h_{2}}^{0} \int_{t+\theta}^{t} \dot{x}^{T}(s) R_{3} \dot{x}(s) d s d \theta \\
& +\int_{-h_{2}}^{-h_{1}} \int_{t+\theta}^{t} \dot{x}^{T}(s) R_{4} \dot{x}(s) d s d \theta
\end{aligned}
$$

where $z(t)=\left[x^{T}(t) x^{T}(t-\boldsymbol{h})\right]^{T}$. This functional corresponds to a classical type of Lyapunov-Krasovskii functionals to check the stability of time-delay systems. Here we only consider the assumption on the positivity of $V$ achieved by the positive definiteness of the matrices $P, Q_{i}, R_{j}$. On the other hand, we introduce an additional functional $\mathcal{V}\left(t, x_{t}\right)$ given by

$$
\begin{aligned}
\mathcal{V}\left(t, x_{t}\right) & =\left(\bar{T}_{k}-\boldsymbol{\tau}\right) \zeta_{1}^{T}(t)\left[S_{1} \zeta_{1}(t)+2 S_{2} z\left(t_{k}\right)\right] \\
& +\left(\bar{T}_{k}-\boldsymbol{\tau}\right) \int_{t_{k}}^{t} \dot{z}^{T}(s) U \dot{z}(s) d s \\
& +\left(\bar{T}_{k}-\boldsymbol{\tau}\right) \boldsymbol{\tau} z^{T}\left(t_{k}\right) X z\left(t_{k}\right),
\end{aligned}
$$

where $\zeta_{1}(t)=z(t)-z\left(t_{k}\right)$. The first step of the proof consists in establishing some properties of the additional functional $\mathcal{V}$. To prove the continuity over $\left[t_{k} t_{k+1}\right]$, note that for $t=t_{k}^{+}$, $\zeta_{0}\left(t_{k}\right)=0$, the integral is zero and the last term is also zero since $\tau\left(t_{k}\right)=0$. When $t=t_{k+1}^{-}, \bar{T}_{k}-\tau$ is equal to zero. Then following equalities are satisfied

$$
\begin{aligned}
& \lim _{\epsilon \rightarrow 0} \mathcal{V}\left(t_{k}-\epsilon, x_{t_{k}-\epsilon}\right)=0, \\
& \lim _{\epsilon \rightarrow 0} \mathcal{V}\left(t_{k}+\epsilon, x_{t_{k}+\epsilon}\right)=0
\end{aligned}
$$

The functional $\mathcal{V}$ is thus continuous with respect to $t$ and is differentiable over $\left[t_{k} t_{k+1}\right.$ [. Thus the functional $V+\mathcal{V}$ is continuous with respect to the time variable and moreover $\mathcal{V}$ is zero at all sampling instants. This makes $\mathcal{V}$ suitable for applying Theorem 1. As suggested in the theorem, no additional constraint is introduced on $S_{1}, S_{2}, U$ and $X$. Thus $V+\mathcal{V}$ is not necessary positive definite within two sampling instants. This corresponds to the improvement with respect to the previous approaches exposed in the case of sampled data systems [Fridman, 2010, Liu and Fridman, 2009a, Seuret, 2009] and [Liu and Fridman, 2009a, Naghshtabrizi et al., 2010, Millán et al., 2009] for sampled and delayed input. Note that the positivity of $U$ is not required as it will be introduced in the sequel.

The rest of the proof consists in ensuring $\mathcal{W}<0$ over $\left[t_{k} t_{k+1}[\right.$. The computation of the derivative of $\mathcal{W}$ leads to

$$
\begin{aligned}
\mathcal{W}\left(t, x_{t}\right. & \left., \dot{x}_{t}\right)=2 z^{T}(t) P \dot{z}(t)+x^{T}(t) Q_{1} x(t) \\
& -x_{t}^{T}\left(h_{1}\right) Q_{12} x_{t}\left(h_{1}\right)-(1-\dot{\boldsymbol{h}}) x_{t}^{T}(\boldsymbol{h}) Q_{23} x_{t}(\boldsymbol{h}) \\
& -x_{t}^{T}\left(h_{2}\right) Q_{3} x_{t}\left(h_{2}\right)-(1-\dot{\boldsymbol{h}}) \dot{x}_{t}^{T}(\boldsymbol{h}) R_{1} \dot{x}_{t}(\boldsymbol{h}) \\
& +\dot{x}^{T}(t) \tilde{R}\left(h_{i}\right) \dot{x}(t)-\zeta_{1}^{T}(t)\left[S_{1} \zeta_{1}(t)+2 S_{2} z\left(t_{k}\right)\right] \\
& +\left(\bar{T}_{k}-\boldsymbol{\tau}\right) \dot{z}(t)^{T}\left[U \dot{z}(t)+2 S_{1} \zeta_{1}(t)+2 S_{2} z\left(t_{k}\right)\right] \\
& +\left(\bar{T}_{k}-2 \boldsymbol{\tau}\right) z^{T}\left(t_{k}\right) X z\left(t_{k}\right) \\
& -\int_{t-h_{2}}^{t} \dot{x}^{T}(s) R_{3} \dot{x}(s) d s-\int_{t-h_{2}}^{t-h_{1}} \dot{x}^{T}(s) R_{4} \dot{x}(s) d s \\
& -(1-\dot{\boldsymbol{h}}) \int_{t-\boldsymbol{h}}^{t} \dot{x}^{T}(s) R_{2} \dot{x}(s) d s-\int_{t_{k}}^{t} \dot{z}^{T}(s) U \dot{z}(s) d s .
\end{aligned}
$$

Introduce the augmented vector $\phi(t) \in \mathbb{R}^{7 n}$ such that

$$
\phi(t)=\left[z^{T}(t) x_{t}^{T}\left(h_{1}\right) x_{t}^{T}\left(h_{2}\right)(1-\dot{\boldsymbol{h}}) \dot{x}_{t}^{T}(\boldsymbol{h}) z^{T}\left(t_{k}\right)\right]^{T} .
$$

It regroups the necessary variables to represents the dynamics of the system. From the definition of the delay $\boldsymbol{h}$ given in (2), and knowing that the matrices $R_{1}$ is positive definite, we have $-1 /(1-\dot{\boldsymbol{h}}) \leq-1 /\left(1-\epsilon_{1}\right)$ and

$$
\begin{aligned}
-(1-\dot{\boldsymbol{h}}) \dot{x}_{t}^{T}(\boldsymbol{h}) R_{1} \dot{x}_{t}(\boldsymbol{h}) & =-1 /(1-\dot{\boldsymbol{h}}) \phi^{T}(t) M_{5}^{T} R_{1} M_{5} \phi(t) \\
& \leq-1 /\left(1-\epsilon_{1}\right) \phi^{T}(t) M_{5}^{T} R_{1} M_{5} \phi(t)
\end{aligned}
$$

Concerning the integrals of (18), they can be rewritten as follows

$$
\begin{aligned}
& -\int_{t-h_{2}}^{t} \dot{x}^{T}(s) R_{3} \dot{x}(s) d s-\int_{t-h_{2}}^{t-h_{1}} \dot{x}^{T}(s) R_{4} \dot{x}(s) d s \\
- & (1-\dot{\boldsymbol{h}}) \int_{t-\boldsymbol{h}}^{t} \dot{x}^{T}(s) R_{2} \dot{x}(s) d s-\int_{t_{k}}^{t} \dot{z}^{T}(s) U \dot{z}(s) d s \\
= & -\int_{t_{k}}^{t} \dot{z}^{T}(s) U \dot{z}(s) d s \\
- & \int_{t-h_{1}}^{t} \dot{x}^{T}(s)\left((1-\dot{\boldsymbol{h}}) R_{2}+R_{3}\right) \dot{x}(s) d s \\
- & \int_{t-h_{1}}^{t} \dot{x}^{T}(s)\left((1-\dot{\boldsymbol{h}}) R_{2}+R_{3}+R_{4}\right) \dot{x}(s) d s \\
& -\int_{t-h_{2}}^{t-h} \dot{x}^{T}(s)\left(R_{3}+R_{4}\right) \dot{x}(s) d s \\
= & I_{1}+I_{2}+I_{3}+I_{4}
\end{aligned}
$$


The following developments concern the integral terms of (19). Consider the last integral and a matrix $\bar{Y}_{3} \in \mathbb{R}^{7 n \times 2 n}$ and the following equality

$$
2 \phi^{T}(t) \bar{Y}_{3}\left[z(t)-z\left(t_{k}\right)\right]=\int_{t_{k}}^{t}\left[2 \phi^{T}(t) \bar{Y}_{3} \dot{z}(s)\right] d s .
$$

Since $U$ is assumed to be positive definite and thus non singular, a classical bounding ensures that for all $t \in\left[t_{k}, t_{k+1}\right.$ [ and for all $s \in\left[t_{k}, t\right]$

$$
2 \phi^{T}(t) \bar{Y}_{3} \dot{z}(s) \leq \phi^{T}(t) \bar{Y}_{3} U^{-1}\left(\bar{Y}_{3}\right)^{T} \phi(t)+\dot{z}^{T}(s) U \dot{z}(s) .
$$

Integrating the previous inequality over $\left[t_{k}, t\right]$, the following inequality is obtained

$$
I_{1} \leq-2 \phi^{T}(t) \bar{Y}_{3}\left(z(t)-z\left(t_{k}\right)\right)+\boldsymbol{\tau} \phi^{T}(t) \bar{Y}_{3} U^{-1} \bar{Y}_{3}^{T} \phi(t),
$$

Since $\dot{h} \leq \epsilon_{2}$ and $R_{2}>0$, applying Jensen's inequality to the first integral of (19) leads to

$$
I_{2} \leq-\phi^{T}(t) M_{13}^{T} \frac{\left(1-\epsilon_{2}\right) R_{2}+R_{3}}{h_{1}} M_{13} \phi(t) .
$$

Based on the same bounding method, the two last integrals of (19) are bounded as follows

and

$$
I_{3} \leq \phi^{T}(t)\left[2 \bar{Y}_{2} M_{23}+\left(\boldsymbol{h}-h_{1}\right) \bar{Y}_{2} \Pi_{32}^{-1} \bar{Y}_{2}^{T}\right] \phi(t)
$$

$$
I_{4} \leq \phi^{T}(t)\left[-2 \bar{Y}_{1} M_{24}+\left(h_{2}-\boldsymbol{h}\right) \bar{Y}_{1} \Pi_{31}^{-1} \bar{Y}_{1}^{T}\right] \phi(t),
$$

where $\bar{Y}_{1}, \bar{Y}_{2} \in \mathbb{R}^{7 n \times n}$. Noting that

$$
\dot{x}(t)=A x(t)+A_{d} x\left(t_{k}-h_{k}\right)=M_{0} \phi(t),
$$

$x(t)=M_{1} \phi(t), \quad x(t-h)=M_{2} \phi(t)$,

$x(t)-x_{t}(h)=M_{12} \phi(t), \quad z(t)=N_{1} \phi(t)$,

$z\left(t_{k}\right)=N_{2} \phi(t), \quad \zeta_{0}(t)=z(t)-z\left(t_{k}\right)=N_{12} \phi(t)$

$\dot{z}(t)=\left[\left(A x(t)+A_{d} x\left(t_{k}-h_{k}\right)\right)^{T}(1-\dot{h}) \dot{x}_{t}^{T}(\boldsymbol{h})\right]^{T}=N_{0} \phi(t)$

Using the definition of the matrices $M_{i}$ 's and $N_{i}$ 's and adding (20), (21) and(22) to (18), the following inequality is obtained for all $t \in\left[t_{k}, t_{k+1}[\right.$

$$
\begin{aligned}
\mathcal{V}(t, & \left.x_{t}, \dot{x}_{t}\right) \leq \phi^{T}(t)\left[\Pi_{1}(\boldsymbol{h})+\left(\bar{T}_{k}-\boldsymbol{\tau}\right) \Pi_{2}\right. \\
& +\left(\bar{T}_{k}-2 \boldsymbol{\tau}\right) N_{2}^{T} X N_{2}+\boldsymbol{\tau} \bar{Y}_{3} U^{-1} \bar{Y}_{3}^{T} \\
& \left.+\left(\boldsymbol{h}-h_{1}\right) \bar{Y}_{2} \Pi_{32}^{-1} \bar{Y}_{2}^{T}+\left(h_{2}-\boldsymbol{h}\right) \bar{Y}_{1} \Pi_{31}^{-1} \bar{Y}_{1}^{T}\right] \phi(t)
\end{aligned}
$$

Applying Lemma 1 with $\lambda(t)=\tau$, the right hand-side term is negative definite if and only if

$$
\begin{aligned}
\Pi_{1}(\boldsymbol{h})+\bar{T}_{k}\left(\Pi_{2}+N_{2}^{T} X N_{2}\right) & +\left(h_{2}-\boldsymbol{h}\right) \bar{Y}_{1} \Pi_{31}^{-1} \bar{Y}_{1}^{T} \\
& +\left(\boldsymbol{h}-h_{1}\right) \bar{Y}_{2} \Pi_{32}^{-1} \bar{Y}_{2}^{T}<0,
\end{aligned}
$$

and

$$
\begin{aligned}
\Pi_{1}(\boldsymbol{h}) & +\bar{T}_{k}\left(N U^{-1} N^{T}-N_{2}^{T} X N_{2}+\bar{Y}_{3} U^{-1} \bar{Y}_{3}^{T}\right) \\
& +\left(h_{2}-\boldsymbol{h}\right) \bar{Y}_{1} \Pi_{31}^{-1} \bar{Y}_{1}^{T}+\left(\boldsymbol{h}-h_{1}\right) \bar{Y}_{2} \Pi_{32}^{-1} \bar{Y}_{2}^{T}<0 .
\end{aligned}
$$

Then noting that the previous conditions depend linearly on $\boldsymbol{h}$, we apply once more Lemma 1 considering $\lambda(t)=\boldsymbol{h}$. The same lemma is finally applied to $\lambda(t)=\bar{T}_{k}$ which belongs to $\left[\max \left\{0, T_{1}+h_{1}-h_{2}\right\}, T_{2}+h_{2}-h_{1}\right]$. The Schur complement allows obtaining conditions (12) and (13). By virtue of Theorem 1, the asymptotically stability of system (5) is guaranteed.

The final step of the proof consists in reducing the number of variables by noting that some variables of the matrices $\bar{Y}_{1}$, $\bar{Y}_{2}$ and $\bar{Y}_{3}$ does not help to solve the conditions of Theorem 2 . Thus we defined the variables $Y_{1}=N_{1} \bar{Y}_{1} \in \mathbb{R}^{2 n \times n}$, $Y_{2}=N_{1} \bar{Y}_{2} \in \mathbb{R}^{2 n \times n}$ and $Y_{3}=N_{3} \bar{Y}_{3} \in \mathbb{R}^{5 n \times 2 n}$

Remark 1. If the minimal bound of the transmission delay $h_{1}$ is zero, it is possible to reduce the size of the LMI and the number of variables to solve the problem. This is because the term $x_{t}\left(h_{1}\right)$ is not required anymore and furthermore, the matrices $Q_{1}$ is then chosen equal to zero.

Remark 2. It is clear that discrete time approaches proposed for instance in [Fujioka, 2009] or in li et al. [2009] leads to less conservative stability conditions. However it is possible to extend the previous stability criteria to the case of timevarying polytopic uncertainties since all the stability conditions provided in this article are linear with respect to the system matrices $A$ and $A_{d}$. This makes the proposed method still relevant with respect to the discrete-time approach.

Remark 3. Note that the conditions of Theorem 2 consider separately the delay parameters $h_{1}, h_{2}, \epsilon_{1}, \epsilon_{2}$ and the sampling period $T$. It is important to see that the conditions require at least the system to be stable with the transmission delay. To see that one can choose $T=0$ and we will obtain the classical LMI conditions for TDS by using Lyapunov-Krasovskii functional.

\section{EXAMPLES}

In this section, the following examples will be studied.

Example 1 Consider system (1) from [Fridman et al., 2004], [Millán et al., 2009], [Naghshtabrizi et al., 2008] with

$$
A=\left[\begin{array}{cc}
0 & 1 \\
0 & -0.1
\end{array}\right], \quad A_{d}=B K=\left[\begin{array}{c}
0 \\
-0.1
\end{array}\right]\left[\begin{array}{l}
-3.75 \\
-11.5
\end{array}\right]^{T} \text {. }
$$

Example 2 Consider system (1) from [Fridman, 2010] with

$$
A=\left[\begin{array}{cc}
-2 & 0 \\
0 & -0.9
\end{array}\right], \quad A_{d}=\left[\begin{array}{cc}
-1 & 0 \\
-1 & -1
\end{array}\right] \text {. }
$$

Example 3 Consider system (1) from [Fridman, 2010] with

$$
\dot{x}(t)=-x\left(t_{k}-h\right) \text {. }
$$

Concerning the time-varying transmission delay, we consider $h_{1}=10^{-4}, \epsilon_{2}=1$ and $\epsilon_{1}=-0.2$ and -1 . The results delivered Theorem 2 for examples 1,2 and 3 are summarized in Table 1 . They show the influence of the delay variation $\epsilon_{1}$. Note finally that the conditions of Theorem 2 with $\bar{T}=0$ are satisfied for example 1. This means that the systems are stable under asynchronous sampling whose maximal periods are given in Table 1 .

\section{CONCLUSION}

A novel analysis of NCS under asynchronous sampling and input delay is provided in this article. This approach is based on the discrete-time Lyapunov Theorem applied to the continuoustime model of the sampled-data systems. Tractable conditions are derived to ensure asymptotic stability. The examples show the efficiency of the method and the reduction of the conservatism compared to other results from the literature. Further work would reduce the conservatism of the stability conditions.

\section{REFERENCES}

Chen, T. and Francis, B. (1995). Optimal sampled-data control systems. Springer-Verlag, Berlin, Germany.

Fridman, E. (2010). A refined input delay approach to sampleddata control. To appear in Automatica.

Fridman, E., Seuret, A., and Richard, J.P. (2004). Robust sampled-data stabilization of linear systems: An input delay approach. Automatica, 40(8), 1141-1446. 


\begin{tabular}{|l|c|c|c|c|c|c|c|}
\hline Ex.1 & & & & & & & \\
\hline$h_{2}$ & $10^{-3}$ & 0.2 & 0.4 & 0.6 & 0.8 & 1 & 1.061 \\
\hline$[$ Naghshtabrizi et al., 2010] & 1.111 & 0.714 & 0.469 & 0.269 & 0.069 & - & - \\
[Millán et al., 2009] & 1.042 & 0.843 & 0.643 & 0.443 & 0.243 & 0.043 & - \\
{$[$ Liu and Fridman, 2009b] } & 1.638 & 1.063 & 0.786 & 0.541 & 0.301 & 0.054 & - \\
\hline Th. 2, $\epsilon_{1}=-0.2$ & 1.671 & 1.307 & 1.034 & 0.76 & 0.462 & 0.121 & $10^{-3}$ \\
Th. 2, $\epsilon_{1}=-1$ & 1.640 & 1.049 & 0.798 & 0.564 & 0.322 & 0.059 & - \\
\hline \hline Ex.2 & & & & & & & \\
\hline$h_{2}$ & $10^{-3}$ & 0.5 & 1 & 1.5 & 2 & 3 & 3.307 \\
\hline$[$ Naghshtabrizi et al., 2010] & 1.278 & 0.499 & - & - & - & - & - \\
{$[$ Millán et al., 2009] } & 1.867 & 1.368 & 0.868 & - & - & - & - \\
{$[$ Liu and Fridman, 2009b] } & 1.970 & 1.368 & 0.868 & 0.443 & 0.212 & 0.038 & - \\
\hline Th. 2, $\epsilon_{1}=-0.2$ & 2.511 & 1.878 & 1.666 & 1.424 & 1.167 & 0.512 & $10^{-3}$ \\
Th. 2, $\epsilon_{1}=-1$ & 2.444 & 1.362 & 1.130 & 0.856 & 0.484 & - & - \\
\hline \hline Ex.3 & & & & & & & \\
\hline$h_{2}$ & $10^{-3}$ & 0.1 & 0.3 & 0.6 & 0.9 & 1.2 & 1.346 \\
\hline$[$ Naghshtabrizi et al., 2010] & 1.278 & 1.064 & 0.704 & 0.399 & 0.099 & - & - \\
{$[$ Millán et al., 2009] } & 1.338 & 1.239 & 1.039 & 0.739 & 0.439 & 0.139 & - \\
{$[$ Liu and Fridman, 2009b] } & 1.945 & 1.548 & 1.230 & 0.864 & 0.522 & 0.168 & - \\
\hline Th. 2, $\epsilon_{1}=-0.2$ & 1.945 & 1.783 & 1.560 & 1.208 & 0.814 & 0.335 & $10^{-3}$ \\
Th. 2, $\epsilon_{1}=-1$ & 1.914 & 1.473 & 1.209 & 0.890 & 0.551 & 0.179 & - \\
\hline
\end{tabular}

Table 1. Maximal sampling period $\bar{T}$ for time-varying transmission delays(Theorem 2 ) for examples 1 , 2 and 3.

Fujioka, H. (2009). Stability analysis of systems with aperiodic sample-and-hold devices. Automatica, 45(3), 771-775.

$\mathrm{Gu}, \mathrm{K}$., Kharitonov, V.L., and Chen, J. (2003). Stability of timedelay systems. Birkhauser.

Hespanha, J., Naghshtabrizi, P., and Xu, Y. (2007). A survey of recent results in networked control systems. Proceedings of the IEEE, 95(1), 138-162.

Hetel, L., Daafouz, J., and Iung, C. (2006). Stabilization of arbitrary switched linear systems with unknown timevarying delays. Automatic Control, IEEE Transactions on, 51(10), 1668-1674.

li, X.G., Çela, A., Niculescu, S.I., and Reama, A. (2009). Some remark on the stability of networked control systems with periodic scheduling. In Proceedings of the European Control Conference. Budapest, Hongary.

Liu, K. and Fridman, E. (2009a). Discontinuous lyapunov functionals for linear systems with sawtooth delays. In Proceedings of the $8^{\text {th }}$ IFAC Workshop on Time-Delay Systems.

Liu, K. and Fridman, E. (2009b). Stability analysis of networked control systems: a discontiuous Lyapunov functional approach. In Joint $48^{\text {th }}$ IEEE Conference on Decision and Control and $28^{\text {th }}$ Chinese Control Conference. Shanghai, China.

Millán, P., Orihuela, L., Vivas, C., and Rubio, F. (2009). Improved delay-dependent stability criterion for uncertain networked control systems with induced time-varying delays. In $1^{\text {rst }}$ IFAC Workshop on Distributed estimation and Control of Networked control Systems, Necsys'09. Venise, Italy.

Mirkin, L. (2007). Some remarks on the use of time-varying delay to model sample-and-hold circuits. IEEE Trans. on Automatic Control, 52(6), 1009-1112.

Naghshtabrizi, P., Hespanha, J., and Teel, A. (2008). Exponential stability of impulsive systems with application to uncertain sampled-data systems. Systems and Control Letters, 57(5), 378-385.

Naghshtabrizi, P., Hespanha, J., and Teel, A. (2010). Stability of delay impulsive systems with application to networked control systems. Trans. of the Inst. of Measurement and Control, Special Issue on Hybrid and Switched Systems. To appear.
Oishi, Y. and Fujioka, H. (2009). Stability and stabilization of aperiodic sampled-data control systems: An approach using robust linear matrix inequalities. In Joint $48^{\text {th }}$ IEEE Conference on Decision and Control and $28^{\text {th }}$ Chinese Control Conference.

Peet, M., Papachristodoulou, A., and Lall, S. (2009). Positive forms and stability of linear time-delay systems. SIAM Journal on Control and Optimization, 47(6), 3227-3258.

Richard, J.P. (2003). Time delay systems: an overview of some recent advances and open problems. Automatica, 39, 16671694.

Seuret, A. (2009). Stability analysis for sampled-data systems with a time-varying period. In $48^{t h}$ IEEE Conference on Decision and Control.

Seuret, A. (2010). A novel stability analysis of sampled-data systems with applications to multi-rate sampling and packet loss. submitted to Automatica.

Yue, D., Han, Q.L., and Lam, J. (2008). Stability and stabilization of nonuniform sampling systems. Automatica, 44(12), 3222-3226.

Zampieri, S. (2008). A survey of recent results in Networked Control Systems. In Proc. $17^{\text {th }}$ IFAC World Congress. Seoul, Korea.

Zhang, W. and Branicky, M. (2001). Stability of networked control systems with time-varying transmission period. In Allerton Conf. Communication, Control, and Computing.

Zhang, W., Branicky, M., and Phillips, S. (2001). Stability of networked control systems. IEEE Control Systems Magazine, (21). 